Article original

\title{
Prédisposition génétique à la néphropathie diabétique: rôle du polymorphisme I/D du gène ACE
}

\author{
Genetic Predisposition to Diabetic Nephropathy: Role of I/D ACE Gene Polymorphism \\ Sabah HANACHI 1,2, Karima SIFI 1,2, Khalida BOUdAOUd ${ }^{3}$, Salima ZEKRI ${ }^{1}$, Sara HOUCHE ${ }^{1}$, Hanane HOUAMEL ', Soumia HASSI- \\ $\mathrm{N}^{1}{ }^{1}$, Karima BENEMBAREK ${ }^{1,2}$, Nouredine ABADI 1,2 \\ ${ }^{1}$ Laboratoire de biochimie CHU Constantine \\ ¿Laboratoire de biologie et génétique moléculaire, faculté de médecine université Salah Boubnider Constantine 3 \\ ${ }^{3}$ Sevice d'endocrinologie CHU Constantine. aboratoire de recherche : systèmes d'information en santé (LABSIS)
}

Auteur correspondant: hanachisabah@yahoo.fr Soumis le 30/03/2020 ; accepté le 29/04/2020; publié le 21/06/20

\section{MOTS CLÉS \\ Polymorphisme, gène ACE, diabète, néphro- pathie diabétique}

\section{Résumé}

Introduction - La néphropathie diabétique est la principale cause de la maladie rénale chronique et représente la complication la plus fréquente et la plus grave du diabète. Sa pathogénie exacte est complexe et noncomplètement élucidée. Plusieurs facteurs et mécanismes contribuent au développement et à l'issue de cette pathologie.

Les objectifs de notre travail sont de déterminer la fréquence du polymorphisme Insertion(I)/Délétion (D) du gène ACE (angiotensin-converting enzyme) chez des patients diabétiques avec et sans néphropathie et d'établir la relation entre ce polymorphisme et la néphropathie diabétique dans une population de l'Est algérien.

Matériel et Méthodes - Nous avons recruté à cet effet, vingt neuf sujets diabétiques avec néphropathie et trente témoins diabétiques sans néphropathie. L'extraction de l'ADN a été réalisée sur du sang frais par la méthode au $\mathrm{NaCl}$ et le polymorphisme I/D du gène ACE a été déterminé par PCR (polymérase Chaine Réaction).

Un consentement éclairé a été obtenu de l'ensemble des participants.

Résultats - La durée moyenne du diabète chez noscas est de 19,21 \pm 9,31ans ; celle des témoins est de 10,67 $\pm 7,66$ ans. Le diabète de type 1 est plus fréquent chez les néphropathes $(72,41 \%)$, chez les témoins la fréquence du diabète de type 2 est plus importante (73.33\%). Les complications macro-angiopathiques sont plus prévalentes chez les néphropathes. De plus l'association de deux ou plusieurs complications est fréquemment retrouvée. Les fréquences des allèles I et $D$ sont respectivement $13,79 \%$ et $86,21 \%$ chez les sujets témoins alors que les fréquences alléliques chez les sujets avec néphropathie sont respectivement $19,64 \%$ et $80,36 \%$.

Conclusion - Aucune association significative entre ce polymorphisme et la néphropathie diabétique n'a été démontrée. 


\section{KEY WORDS}

Polymorphism, ACE gene, diabetes, diabetic nephropathy

\section{Abstract}

Introduction - Diabetic nephropathy is the leading cause of chronic kidney disease and the most common and serious complication of diabetes. Its exact pathogenesis is complex and not completely elucidated. Several factors and mechanisms contribute to the development and the outcome of this pathology.

The objectives of our work are to determine the frequency of the Insertion (I) / Deletion (D) polymorphism of the ACE gene (angiotensin-converting enzyme) in diabetic patients with and without nephropathy and to establish the relationship between this polymorphism and nephropathy diabetic in a population of eastern Algeria.

Patients and methods - To this end, we recruited twenty-nine diabetic subjects with nephropathy and thirty diabetic controls without nephropathy. DNA extraction was performed on fresh blood by the $\mathrm{NaCl}$ method and the I / D polymorphism of the ACE gene was determined by PCR (polymerase chain reaction).

Informed consent was obtained from all participants.

Results - The average duration of diabetes in our patients is $19.21 \pm 9.31$ years; that of the controls is $10.67 \pm 7.66$ years. Type 1 diabetes is more common in nephropaths (72.41\%), in controls the frequency of type 2 diabetes is higher (73.33\%). Macroangiopathic complications are more prevalent in nephropaths. In addition, the association of two or more complications is frequently found. The frequencies of alleles I and D are respectively $13.79 \%$ and $86.21 \%$ in the control subjects while the allelic frequencies in the subjects with nephropathy are respectively $19.64 \%$ and $80.36 \%$.

Conclusion - No significant association between this polymorphism and diabetic nephropathy has been demonstrated.

\section{Introduction}

Le diabète est un groupe de maladies métaboliques caractérisées par une hyperglycémie chronique résultant d'un défaut de sécrétion de l'insuline ou d'un défaut de son action ou de ces deux anomalies associées [1]. L'hyperglycémie chronique est associée à terme à des complications organiques spécifiques touchant particulièrement les yeux, les reins, les nerfs, le cœur et les vaisseaux [2].Selon les dernières estimations de l'organisation mondiale de la santé (OMS) (2016), le nombre de personnes atteintes de diabète est passé de 108 millions en 1980 à 422 millions en 2014 avec une prévalence mondiale chez les adultes de plus de 18 ans qui est passée de 4,7\% en 1980 à 8,5\% en 2014[3]. L'OMS prévoit qu'en 2030, le diabète sera la septième cause de décès dans le monde [4].

La néphropathie diabétique est la principale cause de la maladie rénale chronique et représente la complication la plus courante et la plus grave du diabète. Sa pathogénie exacte est complexe et non élucidée. Plusieurs facteurs contribuent au développement et à l'issue de la néphropathie diabétique. Un diagnostic précoce et une bonne prise en charge peuvent ralentir la progression de la maladie [5].

L'enzyme de conversion de l'angiotensine (ACE) est un composant du Système Rénine Angiotensine, qui peut convertir l'angiotensine I (Ang I) en un peptide actif appelé l'angiotensine II (Ang II)[6].

Le polymorphisme insertion/délétion (I/D) du gène de l'enzyme de conversion de l'angiotensine 1 a été largement étudié. La présence ou l'absence de l'allèle $D$ est associée à une production plus ou moins abondante de l'ACE[7]. Marre et al, étaient les premiers à rapporter une association entre le gène de l'ACE et la néphropathie diabétique [8]. Depuis, de nombreuses études ont été réalisées mais leurs résultats étaient controversés. 
Les objectifs de notre travail sont de déterminer la fréquence du polymorphisme I/D du gène ACE chez des patients présentant un diabète avec et sans néphropathie et d'établir la relation entre ce polymorphisme et la néphropathie diabétique dans une population de l'Est algérien.

\section{Patients et Méthodes}

Notre étude est de type cas témoin. Le recrutement a concerné cinquante neuf sujets répartis en deux groupes, des cas présentant un diabète avec néphropathie $(n=29)$ et des témoins diabétiques sans néphropathie $(n=30)$ sélectionnés parmi les patients admis au service d'endocrinologie du centre hospitalier universitaire $(\mathrm{CHU}) \mathrm{de}$ Constantine et au service de néphrologie de l'établissement hospitalier spécialisé (EHS)Daksi.

Ont été exclus de notre étude les patients ayant eu une transfusion sanguine et les patients ayant un diabète récent $<$ 5ans.

\section{Méthodologie}

Un questionnaire comprenant toutes les données nécessaires a été établi pour toute la population d'étude. Un interrogatoire minutieux a été mené auprès des patients sélectionnés afin de définir les paramètres personnels et familiaux.

Un consentement éclairé a été obtenu de tous les patients et témoins.

Les prélèvements sanguins

Les prélèvements sanguins pour l'étude génétique et le dosage des paramètres biologiques ont été effectués chez des sujets à jeun depuis au moins 12 heures.

L'extraction de l'ADN a été réalisée sur du sang frais par la méthode au $\mathrm{NaCl}$ selon Miller[9].

\section{Les dosages biologiques}

Le dosage des paramètres lipidiques et de la glycémie a été réalisé au niveau du laboratoire de biochimie du $\mathrm{CHU}$ Constantine sur un automate ARCHITECT Ci 8200,ABBOTT,le dosage de la créatinine et des paramètres urinaires ont été réalisés sur ADVIA et l'hémoglobine glyquée a été dosée sur un analyseur D-10 par chromatographie liquide à haute performance (HPLC).

Toutefois, la clairance de la créatinine a été calculée par la formule de Cockroft et Gault.
Le dosage de la micro albuminurie a été réalisé parimmunoturbidimétrie.

La valeur de référence est $30 \mathrm{mg} / \mathrm{l}$, le ratio (micro-albuminurie /créatinine urinaire) a été calculé(valeurs de référence $:<22$ $\mathrm{mg} / \mathrm{g}$ chez l'homme et < $30 \mathrm{mg} / \mathrm{g}$ chez la femme).

L'étude moléculaire a été effectuée au niveau du laboratoire de biologie et génétique moléculaire de la faculté de médecine de Constantine.

La recherche du polymorphisme I/D du gène ACE a été réalisée par PCR (polymérase Chaine Réaction).

Un milieu réactionnel de PCR ou mix d'un volume final de $25 \mu l$ a été préparé.Ce mix comprend des desoxyribonucleotides triphosphates (dNTP à $2 \mathrm{mM}$ ), une enzyme d'amplification in vitro(Taq polymerase à $5 \mathrm{U} / \mu \mathrm{l}$ ), un environnement réactionnel (tampon10X,MgCl2 $50 \mathrm{mM}, \mathrm{H} 20$ ) et deux amorces oligonucléotidiques à $20 \mathrm{pmol} / \mu \mathrm{l}$ chacune dont les séquences sont les suivantes :

\section{- ECA $1 F$ (Forward): 5' GAT GTG GCC ATC ACA TTC GTC AGA T 3' \\ - ECA 1R (Reverse): 5' CTG GAG ACC ACT CCC ATC CTT TCT 3'}

Les conditions d'amplification étaient comme suit : Une dénaturation initiale à $94^{\circ} \mathrm{C}$ pendant 1 minute, suivie de 30 cycles de $\mathrm{PCR}$, comprenant chacun une dénaturation à $94^{\circ} \mathrm{C}$ pendant 30 secondes, une hybridation à $68^{\circ} \mathrm{C}$ pendant $1 \mathrm{mi}$ nute, une élongation à $72^{\circ} \mathrm{C}$ pendant 1 minute et enfin une élongation finale à $72^{\circ} \mathrm{C}$ pendant 8 minutes.

Les fragments amplifiés ont été contrôlés par électrophorèse horizontale sur un gel d'agarose à $2 \%$.

Les différents génotypes ont été déterminés. La présence d'une bande de 490pb correspond au génotype homozygote inséré I/I ; la bande de 190pb correspond au génotype homozygote délété $D / D$ et le génotype hétérozygote I/D est représenté par deux bandes de 190pb et 490pb (figure 1).

Figure 1. Profil électrophorétique des différents génotypes du polymorphisme I/D du gène ACE

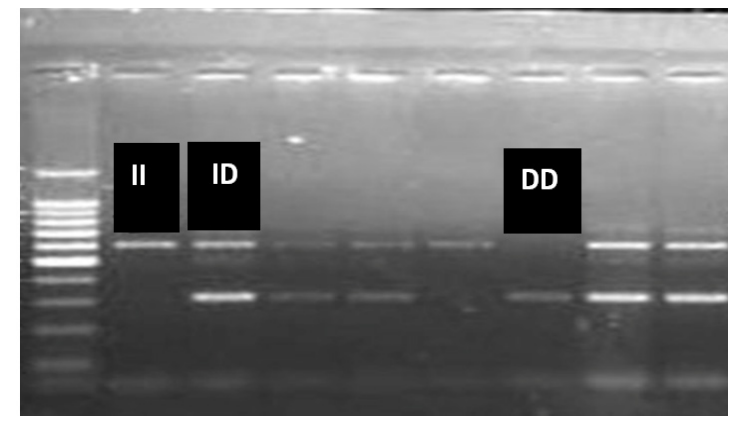


Analyse statistique

L'analyse statistique des données a été effectuée à l'aide du logiciel Epi Info version 7.0.

Le calcul de l'Odds ratio a été utilisé pour déterminer la relation épidémiologique entre le facteur d'exposition et la survenue de la maladie.

L'intervalle de confiance à 95\% est un intervalle de valeurs qui a $95 \%$ de chance de contenir la vraie valeur du paramètre estimé.

Un test de khi 2 a été calculé chez notre population témoin et est revenu à 0,74 avec un Khi 2 test $p$ value égal à 0,39 confirmant que notre population témoins est en équilibre d'Hardy Weinberg.

Le seuil critique a priori est de 0,05 (risque a) .Si la valeur de $p$ calculée est inférieure à ce seuil, la différence entre les paramètres est déclarée statistiquement significative pour le seuil choisi.

\section{Résultats}

L'âge moyen de nos cas est de $58,34 \pm 13,78$ ans ; celui des témoins est de $57,07 \pm 10,51$ ans. La différence d'âge entre les cas et les témoins n'est pas significative ( $P=0,69$ NS).

La répartition de nos cas selon le sexe retrouve treize sujets (soit $44,83 \%$ ) de sexe masculin, et seizesujets (soit 55,17 $\%)$ de sexe féminin. Cette répartition des cas selon le sexe montre que le nombre de cas de sexe féminin est supérieur à celui de sexe masculin avec un sexe ratio $\mathrm{F} / \mathrm{H}$ de 1,23. Chez les témoins, on note également une prédominance féminine avec $66,67 \%$ contre $33,33 \%$

La durée moyenne du diabète chez nos cas est de 19,21 \pm 9,31 ans avec des extrêmes allant de 5 et 45ans; celle des témoins est de 10,67 \pm 7,66 ans. La durée du diabète chez nos cas est significativement plus élevée que chez nos témoins $(p<0,0003)$.

Les fumeurs actifs sont plus prévalents chez les cas $(31,03 \%)$ que chez les témoins $(13,33 \%)$. Les sujets fumeurs sont exclusivement de sexe masculin aussi bien chez les cas que chez les témoins.

Les complications macro-angiopathiques sont plus prévalentes chez les néphropathes dominées par l'hypertension artérielle (HTA). De plus l'association de deux ou plusieurs complications est fréquemment retrouvée (association HTA -artériopathie,HTA-cardiopathie, HTA -artériopathie-cardiopathie....)(Figure 2).
Figure 2 : Fréquence de la macro-angiopathie

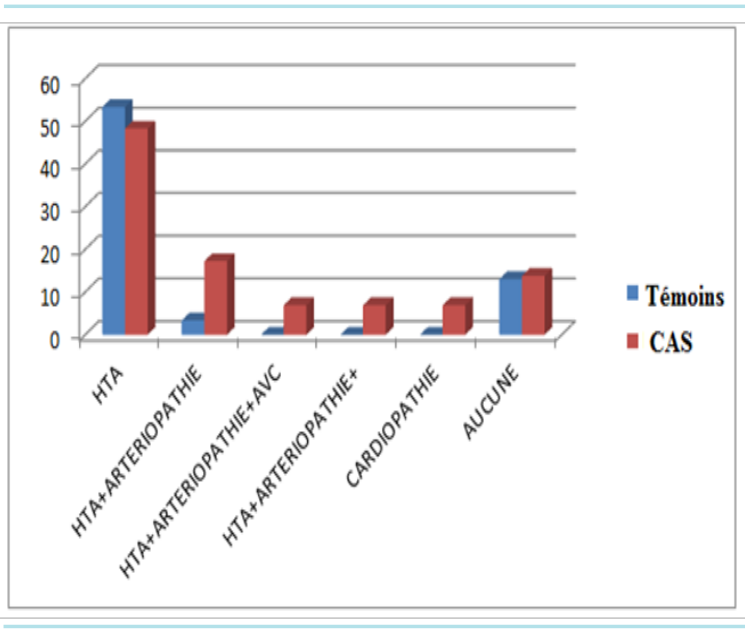

\section{Résultats des paramètres biologiques et génétiques}

Aucune différence significative n'a été retrouvée entre cas et témoins pour tous les paramètres lipidiques.

La comparaison des valeurs moyennes de l'hémoglobine glyquéechez les sujets présentant une néphropathie et les témoins ne montre aucune différence significative.

Fréquences génotypiques et alléliques du polymorphisme I/D du gène $A C E$ chez les cas et les témoins.

La prévalence des différents génotypes du polymorphisme I/D du gène ACEchez les cas est de 64,29\% pour le génotype DD, 32,14 $\%$ pour l'hétérozygotes ID et 3,57 \% pour le génotype II .Chez les témoins, le génotype DD est retrouvé avec une fréquence de $72,41 \%$, la forme hétérozygote ID avec une fréquence de $27,59 \%$ et enfin le génotype homozygote II n'a pas été retrouvé chez nos témoins.

Les fréquences des allèles I et $D$ sont respectivement 13,79 $\%$ et $86,21 \%$ chez les sujets témoins alors que les fréquences alléliques chez les sujets avec néphropathie sont respectivement $19,64 \%$ et $80,36 \%$ (tableau 1 ).

Tableau 1. Fréquences génotypiques et alléliques du polymorphisme I/D du gène ACE chez les cas et les témoins

\begin{tabular}{|l|l|l|l|l|}
\hline $\begin{array}{l}\text { Polymorphisme } \\
\text { I/D du gène } \\
\text { ACE }\end{array}$ & Cas & Témoins \\
\cline { 2 - 5 } & Effectif & $\%$ & Effectif & $\%$ \\
\hline Génotypes & & 3,57 & 0 & 00,00 \\
\hline II & 1 & 32,14 & 8 & 27,59 \\
\hline ID & 9 & 64,29 & 21 & 72,41 \\
\hline DD & 18 & 19,64 & 8 & 13,79 \\
\hline Allèles & 11 & 80,36 & 50 & 86,21 \\
\hline I & 45 & $\begin{array}{l}\text { Intervalle de confiance } \\
0,41-5,20\end{array}$ & $\begin{array}{l}\text { P } \\
=0,71 \text { NS }\end{array}$ \\
\hline D & 1,45 & \multicolumn{5}{|l}{} \\
\hline $\begin{array}{l}\text { Odds ratio(OR) } \\
\text { II+ID vs DD }\end{array}$ & \multicolumn{5}{|l|}{} \\
\hline
\end{tabular}


Le calcul des odds ratios ne montre aucune association entre les génotypes homozygote II et hétérozygote ID versus le génotype homozygote DD.

\section{Discussion}

Dans ce travail, nous nous sommes intéressés à l'étude du polymorphisme I/D du gène ACE chez les diabétiques avec et sans néphropathie à la recherche d'une éventuelle association entre ce polymorphisme et la néphropathie diabétique.

La répartition de nos cas selon le sexe, a montré une prédominance du sexe féminin $(55,17 \%)$ par rapport au sexe masculin $(44,83 \%)$, avec un sexe ratio $F / H$ de 1,23 . Ce résultat ne concorde pas avec les travaux de F.Djrolo et al [10] sur une population de diabétiques noirs africains de Cotonou au Bénin, E. Bouenizabila et al [11]sur une population congolaise de Brazzavilleet V.Viswanathan[12]sur une population sud-indiennequi ont retrouvé une prédominance du sexe masculin.

L'âge moyen de nos cas est proche de celui de VijayViswanathan et al [12]qui était de 56,7+8,9 ans. Les données de la littérature montrent une disparité concernant ce paramètre, certaines retrouvent un âge moyen dans la quarantaine d'autres plutôt au delà de la soixantaine.

La durée du diabète

La durée moyenne du diabète chez nos cas est de 19,21 \pm 9,31 ans ; celle des témoins est de 10,67 $\pm 7,66$ ans. La durée du diabète chez nos cas est significativement plus élevée que chez nos témoins.

En effet, plus la durée d'évolution du diabète est longue plus le diabète est déséquilibré et plus le risque de complication est élevé. Plusieurs études ont confirmé ce résultat $[13,14]$.

Le même résultat est rapporté par plusieurs études telle que l'étude deS.Favaet al [15] l'étude de K .Mansour [16].

L'étude de VijayViswanathan et al [12]retrouve cependant une différence de la durée du diabète non significative entre cas et témoins.

La macro- angiopathie

Les complications macro-angiopathiques sont plus prévalentes chez les néphropathes. De plus l'association de deux ou plusieurs complications est fréquemment retrouvée chez les diabétiques avec néphropathie. L'étude sur une population allemande de Schmidt $S$ et al[17] a retrouvé que les patients diabétiques de type 1 ou 2 ayant eu une néphro- pathie étaient le plus souvent hypertendus et avaient plus fréquemment des événements cardiaques (cardiomyopathie ischémique et / ou cœur congestif.......).

La micro- angiopathie

La fréquence de la rétinopathie est significativement plus élevée chez les cas que chez les témoins ( $79,31 \%$ vs $20,00 \%)$. En effet, dans le diabète de type 2 , comme dans le diabète de type 1 , la rétinopathie et la néphropathie diabétiques sont associées[18].Cependant, les diabétiques de type 2 peuvent présenter d'autres causes de néphropathie que le diabète [19]. L'étiologie diabétique d'une néphropathie pourrait être établie cliniquement devant l'association d'une protéinurie et d'une rétinopathie diabétique. Cette hypothèse se trouve confirmée par une étude de biopsie rénale chez les diabétiques de type 2 avec protéinurie [19].

\section{Paramètres biologiques et génétiques}

L'hémoglobine glyquée est plus élevée chez les cas par rapport aux témoins mais la différence entre les deux groupes reste non significative. Nos résultats s'accordentavec ceux de Y. Wang et al [20]qui ont trouvé un taux de l'hémoglobine glyquée plus élevé chez les cas que chez les témoins $(10,62 \pm 1,93 v s$ 10,93 $\pm 3,54, p=0,519)$ et ceux deM. Pfohl et al [21] qui ont retrouvé le même résultat mais l'élévation était significative $(7,9 \pm 1,4 \mathrm{vs}, 8,4 \pm 1,7 \quad \mathrm{p}=0,04)$.

\section{Polymorphisme I/D du gène ACE}

Les résultats de notre étude montrent que le génotype $D D$ du polymorphisme I/D du gène ACE est non significativement lié à la néphropathie diabétique dans notre population d'étude(OR:1,46 IC (0,41-5,02) p=0,71).

Le rôle du polymorphisme I / $D$ du gène ACEdans la pathogenèse de la néphropathie diabétique a été étudié dans diverses populations mais les résultats restent discordants.

Une méta analyse de $\mathrm{S}$. Schmidt et al[17]n'a retrouvé aucune différence significative de ce polymorphisme entre les Caucasoïdes.

De même, Kumar et al [22]signalent qu'il n'y a pas d'association significative entre le polymorphisme I/D du gène ACE et l'insuffisance rénale diabétique chez les patients diabétiques de type 2 du Nord de l'Inde.

Les étudesdeArfa .I et al [23],Hadjadj .S et al [24],Movva .$S$ et al [25]etErogluZ et al [26]ont montré que dans les populations tunisienne, de race blanche française, indienne et turque respectivement que le polymorphisme I/D du gène ACEn'était pas un marqueur de la néphropathiediabétique et du pronostic rénal des patients atteints de DT2. 
Rahimi Z et al [27]ont montré que les Iraniens d'origine ethnique kurde ne présentent pas une association entre le risque de micro albuminurie et polymorphisme I/D du gène ACE.

Le même résultat a été rapporté par Palomo-Pinon $\mathrm{S}$ et al [28] qui ont montré que les patients mexicains et les témoins ne présentaient aucune différence dans la distribution des génotypes du polymorphisme I/D du gène ACE.

Par ailleurs d'autres études ont retrouvé des résultats discordant avec les nôtres, telle que l'étude deRahimi $Z$ et al[27], qui ont trouvé dans la populationiranienne, une association entre l'allèle $D$ du gène $A C E$ et la néphropathie diabétique

Hadjadj S et al [29]ont montré que dans la population française, le polymorphisme I/D du gène ACE était associé à néphropathiediabétique. En outre, dans une population européenne plus large comprenant des Français, des Danois et des Finlandais, ce polymorphisme était associé de manière significative à un risque accru de néphropathie.

Les études de Nikzamir Aet al $[30,31]$ ont retrouvé que la présence de l'allèle $D$ du gèneACE était associée à une activité plus élevée de l'ACE et à une sévérité accrue de l'albuminurie.

En outre, les résultats d'Ahluwalia TS et al [32] etEzzidi I et al [33]ont montré chez les patients asiatiques, indiens et tunisiens atteints de néphropathie diabétique, que la fréquence de l'allèle $D$ et du génotype DD était significativement plus élevée que chez les patients diabétiques sans néphropathie.

De mêmeDeepak $\mathrm{N}$ et al[34] ont suggéré que le variant $D$ / D dans l'intron 16, contribue à un risque accru de la néphropathie diabétique, mais pas dans l'étendue de sa gravité. Leurs résultats étaient en conformité avec plusieurs autres études [35-39]mais pas toutes [40,41].Cette différence peut être due à la différence ethnique et aux critères de sélection des patients

Notre étude présente cependant des limites dues à la taille réduite de notre échantillon.

\section{Conclusion}

Le génotype $D D$ du polymorphisme I/D du gène ACE ne semble pas associé à la survenue de la néphropathie diabétique dans cet échantillon de diabétiques de l'Est algérien.

La poursuite du présent travail sur un plus grand nombre de patients en vue d'améliorer la puissance des résultats s'avère nécessaire car la démonstration du rôle des facteurs génétiques et environnementaux dans le développement et la progression de la néphropathie diabétique peut ouvrir de nouvelles perspectives dans le diagnostic prédictif et le traitement des patients atteints de cette pathologie et améliorer leur pronostic vital.

\section{Conflits d'intérêt}

Les auteurs déclarent n'avoir aucun conflit d'intérêt.

\section{Références bibliographiques}

[1].Beverly Balkau. Discussion épidémiologique des nouveaux critères de diabète.Médecine thérapeutique / Endocrinologie 2000.volume 2, numero3 : 229. 34.

[2].P. Drouin, J.F. Blickle, B. Charbonnel, E. Eschwege, P.J. Guillausseau, P.F. Plouin, J.M. Daninos, N. Balarac, J.P. Sauvanet . Diagnostic et classification du diabète sucré, les nouveaux critères. Diabetes\&amp; Metabolism, 1999, 1,72.

[3].Mathers CD, Loncar D. Projections of global mortality and burden of disease from 2002 to 2030. PLoS Med, 2006, 3(11): 442.

[4].Organisation Mondiale de la Santé.DIABÈTE.(30/10/2018). Dispônible sur : «https://www.who.int/fr/».

[5].Zhang J, Liu J, X.Qin . Advances in early biomarkers of diabetic nephropathy.Rev Assoc Med Bras ,2018 , 64(1) , 85-92.

[6].Z.Bouchaar, M. Boufrah. Association du polymorphisme I/D de l'enzyme de conversion de l'angiotensine (ECA) et cancer colorectal [mémoire].Constantine : Université des Frères Mentouri, 2017, p 49.

[7].Dogra G, Watts GF. Genes and diabetic nephropathy : what have we learnt so far ? PractDiab Int $2000 ; 17: 84-90$.

[8] Marre M, Bernadet P, Gallois $Y$ et al. Relationships between angiotensin 1 converting enzyme gene polymorphism, plasma levels, and diabetic retinal and renal complications. Diabetes 1994 ; 43 : 384-8.

[9].Miller SA, Dykes DD, Polesky HF. A simple salting out procedure for extracting DNA from human nucleated cells.Nucleicacidsresearch1988;16(3): 1215.

[10].F.Djrolo, V.GatienAttolou, D.GilbertAvode, F.Houngbe, S.Akpona, B.Addra, N.Kodjoh.Néphropathie diabétique :Une étude épidémiologique fondée sur la protéinurie dans une population de diabétiques noirs africains à Cotonou, Bénin. $2001,2,1059$

[11].E. Bouenizabila R. Loumingou , M. Motoula , N. Andzouana , H.-G. Monabeka. La néphropathie diabétique au CHU de Brazzaville, Congo : aspects épidémiologiques, cliniques, et facteurs de risque. Médecine des maladies Métaboliques.2015, 2, 220-226.

[12].V.Viswanathan, Y. Zhu, K.Bala, S. Dunn, C.Snehalatha, A. Ramachandran, M.Jayaraman, K.Sharma.Association between ACE Gene Polymorphism and Diabetic Nephropathy in South Indian Patients.Diabetes Research Centre.2000,4.

[13].El HassaneSidibe. Complications majeures du diabète sucré en Afrique. Ann. Med. Interne, 2000 151, n 8, pp. 624-628. 
[14].Mouton DP, Gill AJ: Prevalence of diabetic retinopathy and evaluation of risk factors: a review of 105 diabetic clinic patients. S Afr Med J, 1988; 74 : 399-402.

[15].S.Fava, J.Azzopardi, S. Ellard,T. Andrew. Hattersley.ACE Gene Polymorphism as a Prognostic Indicator in Patients With Type 2 Diabetes and Established Renal Disease. pathophysiology/Complications.2001,12, 2115-2120.

[16].K. Mansour. Etude des facteurs associés aux complications Chez les diabétiques du RSSB Préfecture des arrondissements de Ben Msik Année 2012.[mémoire]. Epidémiologie de Santé Publique : Ecole Nationale de Santé Publique. (2011-2013), 33 .

[17].Schmidt S, Schöne N, Ritz E.Association of ACE gene polymorphism and diabetic nephropathy.The Diabetic Nephropathy Study Group.Kidney Int. 1995,4, 1176-81.

[18].Insuffisance rénale chronique: étiologies, moyens de diagnostic précoce, prévention? - Paris : INSERM, 1998 - (Expertise collective) ISBN: 2 85598-735-0.

[19].Michel Naricio et al.insuffisance rénale chronique Etiologies, moyens de diagnostic précoce, prévention. 1998,238.

[20]. Wang Y, Peng W, Zhang X, Qiao H, Wang L, Xu Z, Wu C.The association of ACE gene polymorphism with diabetic kidney disease and renoprotective efficacy of valsartan.JReninAngiotensinAldosteroneSyst. $2016,17$.

[21].Pfohl M, Frost D, Koch M, Clemens P, Patzies A, Schmülling RM, Beischer $W$, HäringHU.Lack of association between the insertion/deletion polymorphism of the angiotensin-converting-enzyme gene and diabetic nephropathy in IDDM patients. HormMetabRes. 1998 ,30, 276-80.

[22].Kumar, R, Sharma, RK, Agarwal, S. Genetic predisposition for development of nephropathy in Type 2 diabetes mellitus. Biochem Genet 2013, 51,865-875.

[23].Arfa I, Abid A, Nouira S, Elloumi-Zghal H, Malouche D, Mannai I. et al. Lack of association between the angiotensin-converting enzyme gene (I/D) polymorphism and diabetic nephropathy in Tunisian type 2 diabetic patients. J Renin Angiotensin Aldosterone Syst. 2008,9, 32-6.

[24].Hadjadj S, Fumeron F, Roussel R, Saulnier PJ, Gallois Y, Ankotche A. et al. Prognostic value of the insertion/deletion polymorphism of the ACE gene in type 2 diabetic subjects. Diabetes Care . 2008,31,1847-52.

[25].Movva S, Alluri RV, Komandur S, Vattam K, Eppa K, Mukkavali KK. et al. Relationship of angiotensin-converting enzyme gene polymorphism with nephropathy associated with type 2 diabetes mellitus in Asian Indians. J Diabetes Complications . 2007,21,237-241.

[26].Eroglu Z, Cetinkalp S, Erdogan M. Association of the angiotensinogen M235T and angiotensin-converting enzyme insertion/deletion gene polymorphism in Turkish type 2 diabetic patients with and without nephropathy . J Diabetes Complications. 2008,22, 186-90.

[27].RahimiZ, Felehgari V, Rahimi M, Mozafari H, Yari K, Vaisi-Raygani A. et al. The frequency of factor $V$ Leiden mutation, ACE gene polymorphism, serum ACE activity and response to ACE inhibitor and angiotensin II receptor antagonist drugs in Iranians type II diabetic patients with microalbuminuria. Mol BiolRep . 2011,38,2117-23.

[28].Palomo-Pinon S, Gutierrez-Rodriguez ME, Diaz-Flores M, Sanchez-Barrera $R$, Valladares-Salgado $A$, Utrera-Barillas $D$. et al. DD genotype of angiotensin converting enzyme in type 2 diabetes mellitus with renal disease in Mexican mestizos. Nephrology (Carlton) . 2009,14,235-9.
[29]. Hadjadj S, Tarnow L, Forsblom C, Kazeem G, Marre M, Groop PH. et al. Association between angiotensin-converting enzyme gene polymorphisms and diabetic nephropathy: case-control, haplotype, and family-based study in three European populations. J Am SocNephrol . 2007,18,1284-91.

[30].Nikzamir A, Nakhjavani M, Esteghamati A, Rashidi A. Correlates of ACE activity in macroalbuminuric type 2 diabetic patients treated with chronic ACE inhibition. Nephrol Dial Transplant . 2008,23,1274-7.

[31].Nikzamir A, Esteghamati A, Feghhi M, Nakhjavani M, Rashidi A, Reza JZ. The insertion/ deletion polymorphism of the angiotensinconverting enzyme gene is associated with progression, but not development, of albuminuria in Iranian patients with type 2 diabetes. J Renin-Angiotensin-AldosteroneSyst . 2009,10,109-4.

[32].Ahluwalia TS, Ahuja M, Rai TS, Kohli HS, Bhansali A, Sud K. et al. ACE variants interact with the RAS pathway to confer risk and protection against type 2 diabetic nephropathy. DNA Cell Biol. 2009,28,141-50.

[33].Ezzidil, Mtiraoui N, Kacem M, Chaieb M, Mahjoub T, AlmawiWY.Identification of specific angiotensin-converting enzyme variants and haplotypes that confer risk and protection against type 2 diabetic nephropathy. Diabetes Metab Res Rev. 2009,25,717-24.

[34].Deepak N. Parchwani,Kamlesh M. Palandurkar,D. HemaChandan Kumar ,Darshan J. Patel. Genetic Predisposition to Diabetic Nephropathy: Evidence for a Role of ACE (I/D) Gene Polymorphism in Type 2 Diabetic Population from Kutch Region. Ind J ClinBiochem (Jan-Mar 2015) 30(1):43-54.

[35].Tien KJ, Hsiao JY, Hsu SC, Liang HT, Lin SR, Chen HC, et al. Gender-dependent effect of ACE I/D and AGT M235T polymorphisms on the progres-sion of urinary albumin excretion in Taiwanese with type 2 diabetes. Am J Nephrol. 2009;29: 299-308.

[36].Wang F, Fang Q, Yu N, Zhao D, Zhang Y, Wang J, et al.Association between genetic polymorphism of the angiotensinconverting enzyme and diabetic nephropathy: a meta-analysis comprising 26,580 subjects. J Renin Angiotensin Aldosterone Syst. 2012;13:161-74.

[37].Okuno S, Utsugi T, Ohno T, Ohyama Y, Uchiyama T, Tomono S, et al. Angiotensin-converting enzyme gene polymorphism as a potent risk factor for developing microalbuminuria in Japanese patients with type 2 diabetes mellitus: a 9-year follow-up study. J Int Med Res. 2003;31:290-8.

[38].Ortega-Pierres LE, Gomez Garcla A, Rodriguez-Ayala E, Figueroa-Nunez $B$, Figueroa-Nunez VM, Higareda-Mendoza AE, et al.Angiotensin 1 converting enzyme insertion/deletion genepolymorphism in a Mexican population with diabetic nephropathy. Med Clin (Barc).2007;129:6-10.

[39].Vijay Viswanathan, Yanqing Zhu, KarthikBala, Stephen Dunn, ChamukuttanSnehalatha, Ambady Ramachandran, Muthu Jayaraman, Kumar Sharma. Association between ACE Gene Polymorphism and Diabetic Nephropathy in South Indian Patients.Journal of the Pancreas (Online) 2001; 2(2):83-87.Disponible à l'adressehttp://www.joplink.net .

[40].Jayapalan JJ, Muniandy S, Chan SP. Null association between ACE gene I/D polymorphism and diabetic nephropathy among multiethnic Malaysian subjects. Indian J Hum Genet. 2010;16: 78-86.

[41].Hadjadj S, Gallois Y, Alhenc-Gelas F, Chatellier G, Marre M, Genes N, et al. Angiotensin-I-converting enzyme insertion/deletion polymorphism and high urinary albumin concentration in French type 2 diabetes patients. Diabet Med. 2003;20:677-82. 
